UMD-PP-02-036

\title{
Leptogenesis in models with multi-Higgs bosons
}

\author{
Takeshi Fukuyama円 \\ Department of Physics, Ritsumeikan University, Kusatsu, Shiga 525-8577, Japan \\ and \\ Department of Physics, University of Maryland, College Park, MD 20742, USA
}

Nobuchika Okadaf]

Department of Physics, University of Maryland, College Park, MD 20742, USA

\begin{abstract}
We study the leptogenesis scenario in models with multi-Higgs doublets. It is pointed out that the washing-out process through the effective dimension five interactions, which has not been taken into account seriously in the conventional scenario, can be effective, and the resultant baryon asymmetry can be exponentially suppressed. This fact implies new possible scenario where the observed baryon asymmetry is the remnant of the washed out lepton asymmetry which was originally much larger than the one in the conventional scenario. Our new scenario is applicable to some neutrino mass matrix models which predict too large $\mathrm{CP}$-violating parameter and makes them viable through the washingout process.
\end{abstract}

\footnotetext{
${ }^{1}$ E-Mail: fukuyama@physics.umd.edu

${ }^{2}$ E-Mail: okadan@physics.umd.edu
} 
The origin of the observed baryon asymmetry, the ratio of the baryon density to the entropy density

$$
Y_{B}=\frac{n_{B}-n_{\bar{B}}}{s}=(0.6-1) \times 10^{-10},
$$

is one of the major problems in cosmology. Various scenarios of baryogenesis have been discussed [1], and leptogenesis [2] [3] is one of the attractive scenarios. Providing $U(1)_{B-L}$ breaking in the original theory, lepton number is generated through the CP-violating out-of-equilibrium decay of the right-handed Majorana neutrinos. A part of this lepton number is converted into the baryon number via the $(\mathrm{B}+\mathrm{L})$ violating sphaleron process being in thermal equilibrium 四. As a result, the baryon asymmetry in the universe is generated.

The leptogenesis is also an interesting scenario in the following point of view. Amount of the generated lepton asymmetry (baryon asymmetry) is related to the neutrino Dirac mass matrix, and thus related to neutrino oscillation data [5] through the see-saw mechanism [6] which naturally explains the smallness of the neutrino masses. For detailed discussion, some concrete models are necessary. There have been many works on the models of neutrino mass matrix, where the relationship between the neutrino oscillation data and the observed baryon asymmetry are discussed in detail.

While the conventional leptogenesis scenario has been discussed for one Higgs doublet model, in this letter, we study the leptogenesis in the models with (up-type) multi-Higgs doublets. In multi-Higgs models, one-to-one correspondence between the Yukawa couplings and the neutrino Dirac mass matrix is no longer valid. This fact leads to new possible scenario of the leptogenesis, where the washing-out process through the dimension five interactions plays the crucial role for the resultant baryon asymmetry. Since the effect of this washing-out process has not been considered seriously, the scenario is worth investigating.

Let us first briefly review the conventional leptogenesis scenario. In the following, our discussion is always based on the effective Lagrangian at energies lower than the right-handed neutrino masses such that

$$
\mathcal{L}_{N}=-h_{i j} \overline{l_{L, i}} \phi N_{j}-\frac{1}{2} \sum_{i} \overline{N_{i}^{C}} M_{i} N_{i}+\text { h.c. },
$$

where $i, j=1,2,3$ denote the generation indeces, $h$ is the Yukawa coupling, $l_{L}$ and $\phi$ are the lepton and the Higgs doublets, respectively, and $M_{i}$ is the lepton-number-violating mass term of the right-handed neutrino $N_{i}$ (we are working on the basis of the righthanded neutrino mass eigenstates). For simplicity, we assume the hierarchy among the right-handed neutrino masses, $M_{1} \ll M_{2} \ll M_{3}$, in the following.

The lepton asymmetry in the universe is generated by CP-violating out-of-equilibrium decay of the heavy neutrinos, $N \rightarrow l_{L} \bar{\phi}$ and $N \rightarrow \overline{l_{L}} \phi$. The leading contribution is given 
by the interference between the tree level and one-loop level decay amplitudes, and the $\mathrm{CP}$-violating parameter is found to be [7]

$$
\epsilon=\frac{1}{8 \pi\left(h^{\dagger} h\right)_{11}} \sum_{j} \operatorname{Im}\left[\left(h^{\dagger} h\right)_{1 j}^{2}\right]\left\{f\left(M_{j}^{2} / M_{1}^{2}\right)+2 g\left(M_{j}^{2} / M_{1}^{2}\right)\right\} .
$$

Here $f(x)$ and $g(x)$ correspond to the vertex and the wave function corrections,

$$
\begin{aligned}
f(x) & \equiv \sqrt{x}\left[1-(1+x) \ln \left(\frac{1+x}{x}\right)\right], \\
g(x) & \equiv \frac{\sqrt{x}}{2(1-x)}
\end{aligned}
$$

respectively, and both are reduced to $\sim-\frac{1}{2 \sqrt{x}}$ for $x \gg 1$. We have assumed that the lightest $N_{1}$ decay dominantly contributes to the resultant lepton asymmetry. In fact, this is confirmed by numerical analysis in the case of hierarchical right-handed neutrino masses [8]. Using the above $\epsilon$, the generated $Y_{B}$ is described as

$$
Y_{B} \sim \frac{\epsilon}{g_{*}} d
$$

where $g_{*} \sim 100$ is the effective degrees of freedom in the universe at $T \sim M_{1}$, and $d \leq 1$ is so-called the dilution factor. This factor parameterizes how the naively expected value $Y_{B} \sim \epsilon / g_{*}$ is reduced by washing-out processes.

We can classify the washing-out processes into two cases with and without the external leg of the heavy right-handed neutrinos, respectively. The former includes the inversedecay process and the lepton-number-violating scatterings mediated by the Higgs boson 99 such as $N+\overline{l_{L}} \leftrightarrow \overline{q_{R}}+q_{L}$, where $q_{L}$ and $q_{R}$ are quark doublet and singlet, respectively. The latter case is the one induced by the effective dimension five interaction,

$$
\mathcal{L}_{N} \sim \frac{h^{2}}{M} l l \phi \phi
$$

after integrating out the heavy right-handed neutrinos. Here we have described the interaction symbolically omitting the generation indeces. This term is nothing but the one providing the see-saw mechanism [6]. The importance of this interaction was discussed in [10], where the interaction was shown to be necessary to avoid the false generation of the lepton asymmetry in thermal equilibrium. While numerical calculations [8] [9] are necessary in order to evaluate the dilution factor precisely, $Y_{B} \sim \epsilon / g_{*}$ roughly gives a correct answer, and the washing-out process is mostly not so effective. Note that this is the consequence from the current neutrino oscillation data as explained in the following.

\footnotetext{
${ }^{1}$ Through out this letter, our notations are all based on Ref. [8].
} 
The condition for a washing-out process to be effective is roughly given by

$$
\Gamma\left(T \sim M_{1}\right) \geq H\left(T \sim M_{1}\right) \sim \sqrt{g_{*}} \frac{M_{1}^{2}}{M_{P}}
$$

where $\Gamma$ denotes the decay width or the thermal-averaged cross section times number density for the lepton-number-violating scatterings, $H$ is the Hubble parameter, and $M_{P} \sim 10^{19} \mathrm{GeV}$ is the Planck mass. This condition leads to the lower bound on the light Majorana neutrino mass eigenvalues. $\left(m_{D}^{\dagger} m_{D}\right)_{i i} / M_{i} \sim m_{\nu i}$ through the see-saw mechanism, where $v=174 \mathrm{GeV}$ is the vacuum expectation value (VEV) of the Higgs doublet, and $m_{\nu i}$ is the mass eigenvalue of the light Majorana neutrino in the $i$-th generation.

Considering that the (inverse) decay width is given by

$$
\Gamma_{D} \sim c\left(h^{\dagger} h\right)_{11} M_{1} \sim c m_{\nu 1} M_{1}^{2} / v^{2},
$$

where $c \sim 10^{-2}$ is the phase space factor, we can find $m_{\nu 1} \geq \mathcal{O}\left(10^{-3} \mathrm{eV}\right)$. Although estimation for the Higgs mediated scatterings is much more complicated, we obtain the similar result. To evaluate the condition for the washing-out process due to the dimension five interaction needs knowledge of the flavor structure in the neutrino sector. For simplicity, assume that the dominant contributions are coming from each single $N_{i}$ exchange for $i$ fixed. In this case, we find the cross section

$$
\sigma \sim c \sum_{i}\left(h^{\dagger} h\right)_{i i}^{2} / M_{i}^{2} \sim c \sum_{i} m_{\nu i}^{2} / v^{4}
$$

and the thermal-averaged cross section times number density is given by $\Gamma\left(T \sim M_{1}\right) \sim$ $\sigma M_{1}^{3}$. This leads to the bound such as $\sum_{i} m_{\nu i}^{2} \geq \mathcal{O}\left(\mathrm{eV}^{2}\right)\left(\frac{10^{10} \mathrm{GeV}}{M_{1}}\right)$. Note that this result is incompatible with the neutrino oscillation data [5] in the case of hierarchical light neutrino masses, since $\sum_{i} m_{\nu i}^{2} \sim \Delta m_{\oplus}^{2} \sim 10^{-3} \mathrm{eV}^{2}$, where $\Delta m_{\oplus}^{2}$ is the neutrino oscillation parameter relevant for the atmospheric neutrino anomaly. For the degenerate case it may be allowed, but may have a conflict with the constraint $\sum_{i} m_{\nu i} \leq 1.8 \mathrm{eV}$ due to observations of the large scale structures in the present universe [11]. The possibility $M_{1} \gg 10^{10}$ $\mathrm{GeV}$ would be unlikely in the view point of the re-heating temperature of the inflationary universe. The condition for the inverse-decay can be consistent with the neutrino oscillation data, and can be effective. In this case, there is the useful approximation formula [12]: $d \sim 1 /\left(k(\log k)^{0.6}\right)$ for $k>1$, where $k \sim \Gamma_{D} / H\left(M_{1}\right)$. Considering the relation $d \sim 1 / k \propto 1 / m_{\nu 1}$, the neutrino oscillation data and the cosmological constraint, we can expect $d \geq 10^{-3}$. As a result, both of the washing-out processes are not so important for the resultant baryon asymmetry in the conventional leptogenesis scenario.

Now let us study how the above conclusion is changed in models with the multi-Higgs doublets. For simplicity, we consider the model with two up-type Higgs doublets, $H_{1}^{u}$

\footnotetext{
${ }^{2}$ See Eqs. (24) and (25) for more precise evaluation.
} 
and $H_{2}^{u}$. This simple model includes all the essential points of our discussion. The Dirac neutrino mass matrix is written by the sum of two Higgs doublets $H_{i}^{u}$,

$$
m_{D}=h_{1}\left\langle H_{1}^{u}\right\rangle+h_{2}\left\langle H_{2}^{u}\right\rangle=\left(h_{1} \cos \beta+h_{2} \sin \beta\right) v,
$$

where $v$ and $\beta$ are defined as $v^{2}=\left\langle H_{1}^{u}\right\rangle^{2}+\left\langle H_{2}^{u}\right\rangle^{2}$ and $\tan \beta=\left\langle H_{2}^{u}\right\rangle /\left\langle H_{1}^{u}\right\rangle$, respectively. In the following analysis, we take $v=174 \mathrm{GeV}$ as in the conventional scenario, for definiteness. It is straightforward to extend all the formulas necessary in the leptogenesis scenario to the ones in the multi-Higgs case. For example, the CP-violating parameter Eq. (1) is modified to be the sum of

$$
\epsilon_{a}=\frac{1}{16 \pi\left(h_{a}^{\dagger} h_{a}\right)_{11}} \sum_{b} \sum_{j} \operatorname{Im}\left[\left(h_{a}^{\dagger} h_{b}\right)_{1 j}\left(h_{b}^{\dagger} h_{a}\right)_{1 j}+2\left(h_{a}^{\dagger} h_{a}\right)_{1 j}\left(h_{b}^{\dagger} h_{b}\right)_{1 j}\right] \frac{M_{j}}{M_{1}}
$$

for $M_{1} \ll M_{j}$, where the first (second) term corresponds to the vertex (wave function) correction. The crucial modification in the multi-Higgs models is that the direct relation between the Yukawa couplings and the Dirac mass matrix to be compared with the experimental data is no longer valid, because of the new parameter $\beta$ introduced. Even if the mass matrices, $h_{1}\left\langle H_{1}^{u}\right\rangle$ and $h_{2}\left\langle H_{2}^{u}\right\rangle$, are fixed by some predictive model and/or experiments, there is still freedom to change the Yukawa couplings according to $\beta$. Since general discussion about the multi-Higgs case is very complicated, we assume that $h_{1}\left\langle H_{1}^{u}\right\rangle \ll h_{2}\left\langle H_{2}^{u}\right\rangle$ and $h_{1} \ll h_{2}$ to make the essential point of our discussion clear. These assumption reduces our formulas to the one in the conventional scenario with identification $h=h_{2}$. However, note that the quantity defined as $\bar{m}_{D}=h v$ is not the Dirac mass to be compared with the experiments, and no longer suffer from the neutrino oscillation data. To avoid confusion, we use the notation, $\left(h^{\dagger} h\right)_{i i} v^{2} / M_{i}=\left(\bar{m}_{D}^{\dagger} \bar{m}_{D}\right)_{i i} / M_{i} \sim \bar{m}_{\nu i}$, in the following. Note again that $\bar{m}_{\nu_{i}}$ is not the physical mass eigenvalue.

In the conventional scenario, the direct correspondence between the Yukawa coupling and the Dirac mass matrix allows us to describe the CP-violating parameter as

$$
\epsilon=\frac{3}{16 \pi\left(m_{D}^{\dagger} m_{D}\right)_{11}} \sum_{j} \operatorname{Im}\left[\left(m_{D}^{\dagger} m_{D}\right)_{1 j}^{2}\right] \frac{M_{j}}{M_{1}} .
$$

by using the Dirac mass matrix. Suppose that $m_{D}$ is fixed by some mass matrix models and/or experiments, we can evaluate $\epsilon$. The resultant $\epsilon$ and $Y_{B} \sim \epsilon / g_{*}$ may reveal a large discrepancy against the observed baryon asymmetry. However, note that, in our case, the above formula is modified as $\epsilon \rightarrow \epsilon / \sin ^{2} \beta$ through $m_{D} \rightarrow \bar{m}_{D}=m_{D} / \sin \beta$. Therefore, in the case with too small $\epsilon$, we can easily reproduce the observed baryon asymmetry by adjusting small $\sin \beta$ appropriately. This is the straightforward consequence according to the introduction of new free parameter $\beta$. On the other hand, if $\epsilon$ is too large, there seems to be no hope to obtain the baryon asymmetry consistent with the observation. However, we will show that, in this case, the washing-out processes can play an important role so as to reduce the resultant $Y_{B}$ to the observed values. Since we no longer suffer 
from the neutrino oscillation data, the Yukawa coupling $h=h_{2}$ can be taken to be large enough for the washing-out process to become very effective. This is a new scenario in the leptogenesis which has neither been considered seriously nor be able to be realized in the conventional scenario because of the constraints from the neutrino oscillation data.

Now let us discuss the washing-out processes in detail by integrating the Boltzmann equations out. In the conventional scenario, numerical analysis have been done in detail [9] [8], and all the quantities we need in this analysis are collected there. Thus, we do not discuss about derivations of formulas etc. in this letter. All our formulas are based on Ref. [8]. We are interested in the parameter region where the washing-out processes are effective. Although the conventional analysis has been performed also on this parameter region, it has not been seriously taken into account, because of the incompatibility with the neutrino oscillation data as discussed above.

The Boltzmann equations are written by [8]

$$
\begin{aligned}
\frac{d Y_{N_{1}}}{d z} & =-\frac{z}{s H\left(M_{1}\right)}\left(\frac{Y_{N_{1}}}{Y_{N_{1}}^{e q}}-1\right) \gamma_{D}, \\
\frac{d Y_{B-L}}{d z} & =-\frac{z}{s H\left(M_{1}\right)}\left[\left\{\epsilon\left(\frac{Y_{N_{1}}}{Y_{N_{1}}^{e q}}-1\right)+\frac{1}{2} \frac{Y_{B-L}}{Y_{l}^{e q}}\right\} \gamma_{D_{1}}+2 \frac{Y_{B-L}}{Y_{l}^{e q}}\left(\gamma_{N}+\gamma_{N t}\right)\right],
\end{aligned}
$$

where $z=M_{1} / T$, and $s$ is the entropy density. Here $\gamma_{D}$ is given by

$$
\gamma_{D} \equiv n_{N_{1}}^{e q} \frac{K_{1}(z)}{K_{2}(z)} \Gamma_{D}
$$

with the usual decay width of $N_{1}$ in the rest system,

$$
\Gamma_{D}=\frac{\left(h^{\dagger} h\right)_{11}}{8 \pi} M_{1} \sim \frac{\bar{m}_{\nu 1}}{8 \pi} \frac{M_{1}^{2}}{v^{2}},
$$

the modified Bessel functions $K_{i}$, and the number density $n_{N_{1}}^{e q}=M_{1}^{3} /\left(\pi^{2} z\right) K_{2}(z)$. Here $\gamma_{N}\left(\gamma_{N t}\right)$ corresponds to the lepton-number-violating scattering process $l \phi \leftrightarrow l \phi(l l \leftrightarrow$ $\phi \phi)$ through the effective dimension five interaction of Eq. (4). We have omitted the Higgs mediated processes for simplicity. These processes have an effect similar to that of the inverse-decay, since both are classified in the same washing-out process with the $N_{1}$ external leg and have amplitudes proportional to $\left(h^{\dagger} h\right)_{11}$. For two body scattering, $\gamma$ is given by

$$
\gamma(a, b \leftrightarrow i, j)=\frac{T}{64 \pi^{4}} \int_{\left(m_{a}+m_{b}\right)^{2}}^{\infty} d s \hat{\sigma}(s) \sqrt{s} K_{1}\left(\frac{\sqrt{s}}{T}\right),
$$

with the reduced cross section $\hat{\sigma}(s)$. Assuming, again, that the dominant contribution is due to the single $N_{i}$ exchange for fixed $i$, the sum of the reduced cross section for the scattering $l \phi \leftrightarrow l \phi$ and $l l \leftrightarrow \phi \phi$ is given by

$$
\hat{\sigma}_{N+N t}(s)=\hat{\sigma}_{N}(s)+\hat{\sigma}_{N t}(s) \sim \frac{3}{2 \pi} \sum_{j} \frac{\left(\bar{m}_{D}^{\dagger} \bar{m}_{D}\right)_{j j}^{2}}{v^{4}} \frac{s}{M_{j}^{2}} \sim \frac{3}{2 \pi} \frac{\bar{m}_{\nu 3}^{2}}{v^{4}} s .
$$


Here, we have further assumed the hierarchy $\bar{m}_{\nu 1,2} \ll \bar{m}_{\nu 3}$ for simplicity. This also means that the washing-out process mediated by $N_{3}$ is dominant. Substituting the reduced cross section into Eq. (15), we obtain

$$
\gamma_{N+N t}=\gamma_{N}+\gamma_{N t} \sim \frac{3}{4 \pi} \frac{\bar{m}_{\nu 3}^{2} M_{1}^{6}}{v^{4}} z^{-6}
$$

This result is a good approximation for the region $M_{Z} \ll T \leq M_{1} \ll M_{3}$ which we are interested in, where $M_{Z}$ is the Z-boson mass.

Now we are ready to numerically analyze the Boltzmann equations. Given $\epsilon, \bar{m}_{\nu 1}$ and $\bar{m}_{\nu 3}$, the Boltzmann equations can be easily integrated out. For very small $\bar{m}_{\nu 1}$ and $\bar{m}_{\nu 3}$, we can find $Y_{B-L} \sim \epsilon / g_{*}$. Our interest is on the parameter region where the washing-out processes play the important roles. Now we are free from the neutrino oscillation data, and can take large input values for $\bar{m}_{\nu 1}$ and $\bar{m}_{\nu 3}$. Let us consider two spacial cases. One is that the washing-out process through the inverse-decay is dominant. This case has been taken into account in the previous works with the range of the resultant dilution factor $d \geq 10^{-3}$. The other is that the resultant $Y_{B-L}$ is dramatically reduced by the washing-out through the dimension five interactions. For each cases, $Y_{B-L}$ is depicted in Fig. 1 and Fig. 2, respectively, as a function of $\log _{10}(z) . f^{3}$ Here, we have taken the input parameter $\epsilon=10^{-3}$ as an example. The resultant dilution factor $d$ is independent of this input in this numerical analysis. We can see that there is the dramatic reduction of the resultant baryon number in each graph.

There are useful approximation formulas for two cases discussed above. The Boltzmann equation of Eq. (12) can be rewritten as

$$
\frac{d Y_{B-L}}{d z}+P(z) Y_{B-L}=Q(z)
$$

where

$$
P(z)=\frac{z}{s H\left(M_{1}\right) Y_{l}^{e q}}\left(\frac{1}{2} \gamma_{D}+2 \gamma_{N+N t}\right) \equiv P_{D}(z)+P_{N}(z)
$$

with

$$
\begin{aligned}
P_{D}(z) & =\frac{\bar{m}_{\nu 1} M_{1}^{2}}{32 \pi H\left(M_{1}\right) v^{2}} z^{3} K_{1}(z) \\
P_{N}(z) & =\frac{3 \bar{m}_{\nu 3}^{2} M_{1}^{3}}{4 \pi^{3} H\left(M_{1}\right) v^{4}} z^{-2}
\end{aligned}
$$

and

$$
Q(z)=-\epsilon \frac{z}{s H\left(M_{1}\right)}\left(\frac{Y_{N_{1}}}{Y_{N_{1}}^{e q}}-1\right) \gamma_{D} .
$$

\footnotetext{
${ }^{3}$ The region of the Yukawa couplings in the first case is limited. For large Yukawa couplings, the washing-out process through the dimension five interactions usually becomes important, and dominates (see Eqs.(27) and (28), and compare them).
} 
Here we have used $s Y_{l}^{e q}=\frac{2}{\pi^{2}} M^{3} z^{-3}$. The solution of the above Boltzmann equation with the initial condition $Y_{B-L}(0)=0$ is given by

$$
Y_{B-L}(z)=\int_{0}^{z} d x Q(x) e^{-\int_{x}^{z} P(y) d y}
$$

with the solution of Eq. (11). We can find that $Y_{B-L}(\infty) \sim \epsilon / g_{*}$ if $P(z) \ll 1$.

Noting that the out-of-equilibrium decay begins roughly at $T \sim M_{1}$, the condition $P(1) \geq 1$ gives a reasonable condition for the washing-out processes to be effective. For the above two cases, we can obtain the conditions on the light neutrino mass eigenvalues such that

$$
\begin{aligned}
& P_{D}(1) \geq 1 \quad \rightarrow \quad \bar{m}_{\nu 1} \geq 4 \times 10^{-3} \mathrm{eV}, \\
& P_{N}(1) \geq 1 \quad \rightarrow \quad \bar{m}_{\nu 3} \geq 2 \mathrm{eV}\left(\frac{10^{10} \mathrm{GeV}}{M_{1}}\right)^{1 / 2} .
\end{aligned}
$$

These results are in good agreement with the numerical calculations as can be seen in Fig 1 and 2.

The washing-out processes play the important roles in the case $P(1) \gg 1$. Using the method of the steepest decent in Eq. (23), we can find [12]

$$
Y_{B-L}(\infty) \sim \frac{\epsilon}{g_{*}} a^{-1 / 2} z_{f}^{3 / 2} \exp \left[-z_{f}-\int_{z_{f}}^{\infty} d z P(z)\right],
$$

where $a=-d P(z) /\left.d z\right|_{z=z_{f}}(>0)$ with $z_{f}$ defined as $P\left(z_{f}\right)=1$. In the first case, $P_{D}(1) \gg 1$ but $P_{N}(1) \ll 1$, it is difficult to obtain a simple formula because of the Bessel function in $P_{D}(z)$. Although we can find a rough formula $Y_{B-L} \propto 1 / k$, more reasonable fitting formula is known such as [12]

$$
Y_{B-L}(\infty) \sim \frac{\epsilon}{g_{*}} \times \frac{0.1}{k(\ln k)^{0.6}}
$$

with $k \sim P_{D}(1)$. In the other case, $P_{D}(1) \ll 1$ but $P_{N}(1) \gg 1$, analytic calculation is possible by using Eq. (21), and the approximation formula is found to be

$$
Y_{B-L}(\infty) \sim \frac{\epsilon}{g_{*}} \times \Delta \exp (-2 \sqrt{\Delta})
$$

with

$$
\Delta=\frac{3 \bar{m}_{\nu 3}^{2} M_{1}^{3}}{4 \pi^{3} H\left(M_{1}\right) v^{4}}
$$

We can check that the both formulas give good approximations compared to the numerical results. 
We have learned that there is the parameter region where the washing-out processes become important. Here, remember that $\epsilon$ is the function of the Yukawa couplings and becomes large according to the power law, $\epsilon \propto h^{2}$. In the case $P_{D}(1) \gg 1$ but $P_{N}(1) \ll 1$, from Eq. (27), we can expect that the resultant baryon number is saturated to a constant, as Yukawa coupling becomes large. On the other hand, in the case $P_{N}(1) \gg 1$ but $P_{D}(1) \ll 1$, Eq. (28) leads to the conclusion that the resultant baryon number becomes an exponentially decreasing function of the (large) Yukawa couplings. This implies that, even if the parameter $\epsilon / g_{*}$ itself is too large to be consistent with the observation, new parameter region of large Yukawa couplings comes out, so that the observed baryon number can be reproduced. This is a new interesting scenario.

In summary, we have studied the leptogenesis scenario in models with multi-Higgs doublets. In our scenario, the direct correspondence between the Yukawa coupling and the mass matrix is lost. As a result, new parameter region can be consistent with the neutrino oscillation data. We have pointed out the importance of the washing-out process which has not been seriously taken into account in the conventional scenario. We found a new parameter region of the large Yukawa couplings which can reproduce the observed baryon number through the exponential dumping of too large CP-violating parameter $\epsilon$ due to the washing-out processes through the dimension five interactions .

Finally, we give a comment on the application of our scenario to some neutrino mass matrix models. Many models of the Majorana neutrinos with the see-saw mechanism have been proposed, which predict and/or reproduce the observed neutrino properties. When the leptogenesis scenario is taken into account in such models, some of them may predict too large baryon asymmetry to be consistent with the observations. This is very problematic in cosmology. However, if such a model is the multi-Higgs doublet model, the scenario we have discussed in this letter is applicable and can make the model viable. Further discussion needs a concrete model, and such a model is worth investigating.

The authors would like to thank Rabindra Mohapatra and Markus Luty for useful discussions.

\footnotetext{
${ }^{4}$ If we assume that the re-heating temperature of the inflationary universe is lower than the righthanded neutrino masses, this problem can be easily avoided. However, the leptogenesis no longer works.
} 


\section{References}

[1] For a recent review, see A. Riotto, hep-ph/9807452, and the references therein.

[2] M. Fukugita and T. Yanagida, Phys. Lett. B 174, 45 (1986).

[3] For a recent review, see W. Buchmuller and M. Plumacher, Int. J. Mod. Phys. A 15, 5047 (2000), and the references therein.

[4] V.A. Kuzmin, V.A. Rubakov, and M.E. Shaposhnikov, Phys. Lett. B 155, 36 (1985).

[5] Particle Data Group, D.E. Groom et al., Eur. Phys. J. C 15, 1 (2000).

[6] T. Yanagida, in Proceedings of the workshop on the Unified Theory and Baryon Number in the Universe, edited by O.Sawada and A.Sugamoto (KEK, Tsukuba, 1979); M. Gell-Mann, P. Ramond, and R. Slansky, in Supergravity, edited by D.Freedman and P.van Niewenhuizen (north-Holland, Amsterdam 1979); R.N. Mohapatra and G. Senjanovic, Phys. Rev. Lett. 44, 912 (1980).

[7] L. Covi, E. Roulet, and F. Vissani, Phys. Lett. B 384, 169 (1996); W. Buchmuller and M. Plumacher, Phys. Lett. B 431, 354 (1998).

[8] M. Plumacher, Z. Phys. C 74, 549 (1997).

[9] M.A. Luty, Phys. Rev. D 45, 455 (1992).

[10] E.W. Kolb and S. Wolfram, Nucl. Phys. B 172, 224 (1980); J.N. Fry, K.A. Olive, and M.S. Turner, Phys. Rev. D22, 2953 (1980).

[11] M. Fukugita, G.C. Liu, and N. Sugiyama, Phys. Rev. Lett. 84, 1082 (2000).

[12] E.W. Kolb and M.S. Turner, The Early Universe, Addison-Wesley Publishing Co., California (1990) 


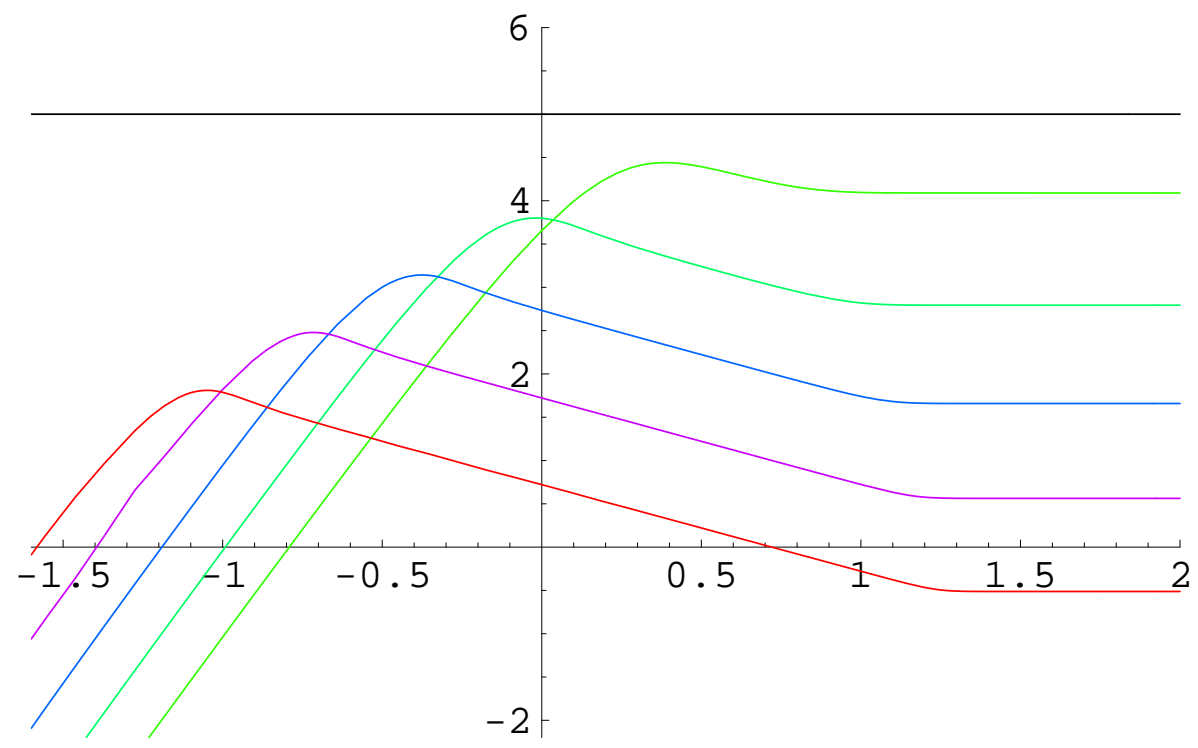

Figure 1: The solution of the Boltzmann equation $\left(\log _{10}\left(Y_{B-L} \times 10^{10}\right)\right)$ with $\epsilon / g_{*}=$ $10^{-5}$ (the upper horizontal line), $M_{1}=10^{10} \mathrm{GeV}$ and $\bar{m}_{\nu 3}=0.2 \mathrm{eV}$. The solutions for $\bar{m}_{\nu 1}=4 \times 10^{-3}, 0.04,0.4,4$ and $40 \mathrm{eV}$ are plotted from above at $z=10^{2}$.

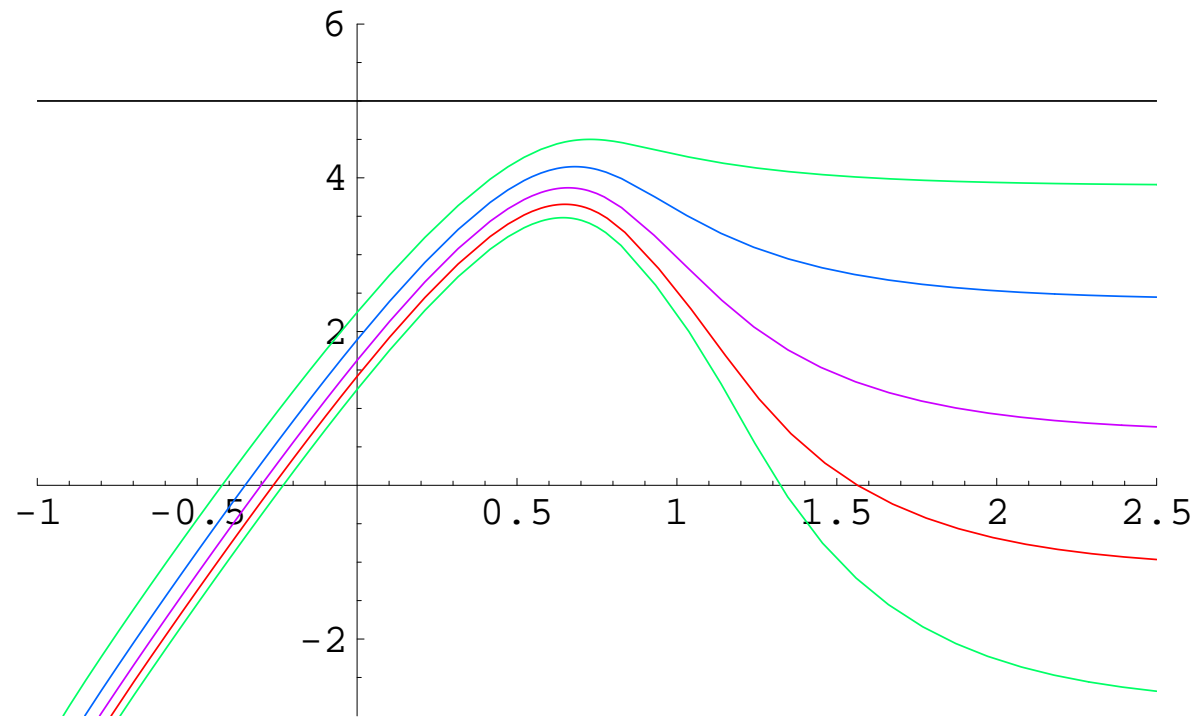

Figure 2: The solution of the Boltzmann equation $\left(\log _{10}\left(Y_{B-L} \times 10^{10}\right)\right)$ with $\epsilon / g_{*}=10^{-5}$, $M_{1}=10^{10} \mathrm{GeV}$ and $\bar{m}_{\nu 1}=4 \times 10^{-4} \mathrm{eV}$. The solutions for the input values $\bar{m}_{\nu 3}=7,12$, 17,22 and $27 \mathrm{eV}$ are plotted from above. 